2 This manuscript is a preprint and has been submitted for publication in Geophysical Journal

3 International. Please note that, despite having undergone peer review, the manuscript has yet to be

4 formally accepted for publication. Subsequent versions of this manuscript may have slightly different

5 content. If accepted, the final version of this manuscript will be available via the 'Peer-reviewed

6 Publication DOI' link on the right-hand side of this webpage. Please feel free to contact any of the

7 authors; we welcome feedback. 


\section{Bayesian differential moment tensor inversion: theory and application to the North Korea nuclear tests}

Zhe Jia, Zhongwen Zhan, Donald Helmberger ${ }^{\dagger}$

Seismological Laboratory, California Institute of Technology, Pasadena, CA 91125, USA

${ }^{\dagger}$ Deceased on August 13, 2020

Corresponding author. Zhe Jia (zjia@gps.caltech.edu)

\section{SUMMARY}

Moment tensors are key to seismic discrimination but often require accurate Green's functions for estimation. This limits the regions, frequency bands, and wave types in moment tensor inversions. In this study, we propose a differential moment tensor inversion (diffMT) method that uses relative measurements to remove the path effects shared by clustered events, thereby improving the accuracy of source parameters. Using results from regular inversions as a priori distribution, we apply Bayesian Markov Chain Monte Carlo to invert the body- and surface-wave amplitude ratios of an event pair for refined moment tensors of both events. Applications to three North Korea nuclear tests from 2013 to 2016 demonstrate that diffMT reduces the uncertainties substantially compared with the traditional waveform-based moment tensor inversion. Our results suggest high percentages of explosive components with similar double-couple components for the North Korea nuclear tests.

Key words: Earthquake source observations, Inverse theory, Earthquake monitoring and test-ban treaty verification 


\section{INTRODUCTION}

Seismic moment tensor provides a point-source approximation of the radiation pattern and a measure of the event size. Different combinations of isotropic (ISO), double couple (DC), and compensated linear vector dipole (CLVD) components can manifest the first-order physics of different event types, such as natural earthquakes, collapses, landslides, and nuclear explosions, thus being used for their discrimination (Alvizuri and Tape, 2018, Ford et al., 2009, Cesca et al., 2017). Furthermore, doublecouple focal mechanisms provide important insights on regional stress state (Hauksson, 1994, Hardebeck and Hauksson, 2001, Wang and Zhan, 2020b), plate interface morphology (Hayes et al., 2009, Bazargani et al., 2013, Zhan et al., 2012) and slab dynamics (Yang et al., 2017, Liu et al., 2021). In the past few decades, moment tensor inversion has gradually progressed from polaritybased to waveform-based inversion (Ekström et al., 2012, Kanamori and Rivera, 2008, Zhu and Helmberger, 1996). At the theoretical level, Tape and Tape $(2012,2013,2015)$ proposed a mathematically intuitive way to view the moment tensors and examine the explosive and tensile mechanisms. Zhu and Ben-Zion (2013) developed a parameterization of full moment tensors with well-defined parameters for source inversion. These progresses in theory and inversion, together with the improving Earth structural modeling, reduce the focal mechanism errors to about 20 degrees for most moderate to large events in the centroid moment tensor catalogs (Duputel et al., 2012).

However, accurate full moment tensor inversions for shallow sources are still challenging. Robust moment tensor solutions are usually only retrievable at long periods (e.g. T>20s, (Minson and Dreger, 2008)) that are insensitive to small-scale structural heterogeneities. However, earthquakes and explosions of small to moderate size usually have limited near-field coverage and weak signals 
at long periods. For the short-period waves, modeling them is difficult because existing 3D crustal velocity models are often inadequate in capturing small scale heterogeneities at regional distances. Using inaccurate earth structural models could introduce errors in focal mechanisms and non-DC proportions (Frohlich and Davis, 1999). Taking the North Korea nuclear explosions as an example, different studies show nontrivial differences of moment tensor solutions (Cesca et al., 2017, Chiang et al., 2018, Alvizuri and Tape, 2018). For better azimuthal and take-off angle coverage, approaches that jointly invert regional and teleseismic waves have been proposed (Ni et al., 2010, Ford et al., 2012), but they still encounter difficulties from inaccurate Green's functions.

To accurately determine the moment tensors when the path structure is complex, approaches using 3D Green's functions have been introduced (Covellone and Savage, 2012, Wang and Zhan, 2020a). Most models used to calculate the 3D Green's function are travel-time- and waveform- based tomographic models. Travel time tomographic models, such as the LLNL model by Simmons et al. (2012) and the SALSA3D model by Ballard et al. (2016), can predict body waves arrivals with significantly reduced errors than 1D models, thereby being used to precisely detect and locate small seismic events. However, they are usually restricted by the smoothing in the inversions, and may not accurately fit the seismic waveforms. On the other hand, waveform-based tomographic models are more promising in explaining wiggles on seismograms (Tape et al., 2009, Fichtner et al., 2009, Bozdağ et al., 2016). But most global and continental scale models use long-period waveforms (e.g. $\mathrm{T}>17 \mathrm{~s}$ globally) for inversion, due to the high computational cost. Only for specific areas of dense seismic monitoring, adjoint tomographic inversions based on higher frequency seismic waveforms have been developed and implemented in source inversions (Savage et al., 2014, Lee et al., 2014, Jia 
et al., 2020b).

To reduce the requirement of highly accurate velocity models, the empirical Green's function (EGF) methods are developed to study clustered explosions and earthquakes. Sites of artificial explosions are often clustered and share similar path and site effects. For example, all the North Korea nuclear tests were in the Punggye-ri site, within a few km from each other (Zhang and Wen, 2013, Zhang and 
Bostock (2019) used relative amplitudes of body waves among a cluster of seismic events to improve focal mechanisms. EGF methods greatly reduce moment tensor errors, but they can also introduce bias by assuming the reference event is well resolved. Dahm (1996) avoided the assumption on a single reference event by using arbitrary a priori constraint, but facing the issues of interference bias and lack of uncertainty assessments. To better assess errors and to avoid arbitrary selection of reference events, we need to incorporate Bayesian statistics to the relative moment tensor inversion methods with appropriate a priori information.
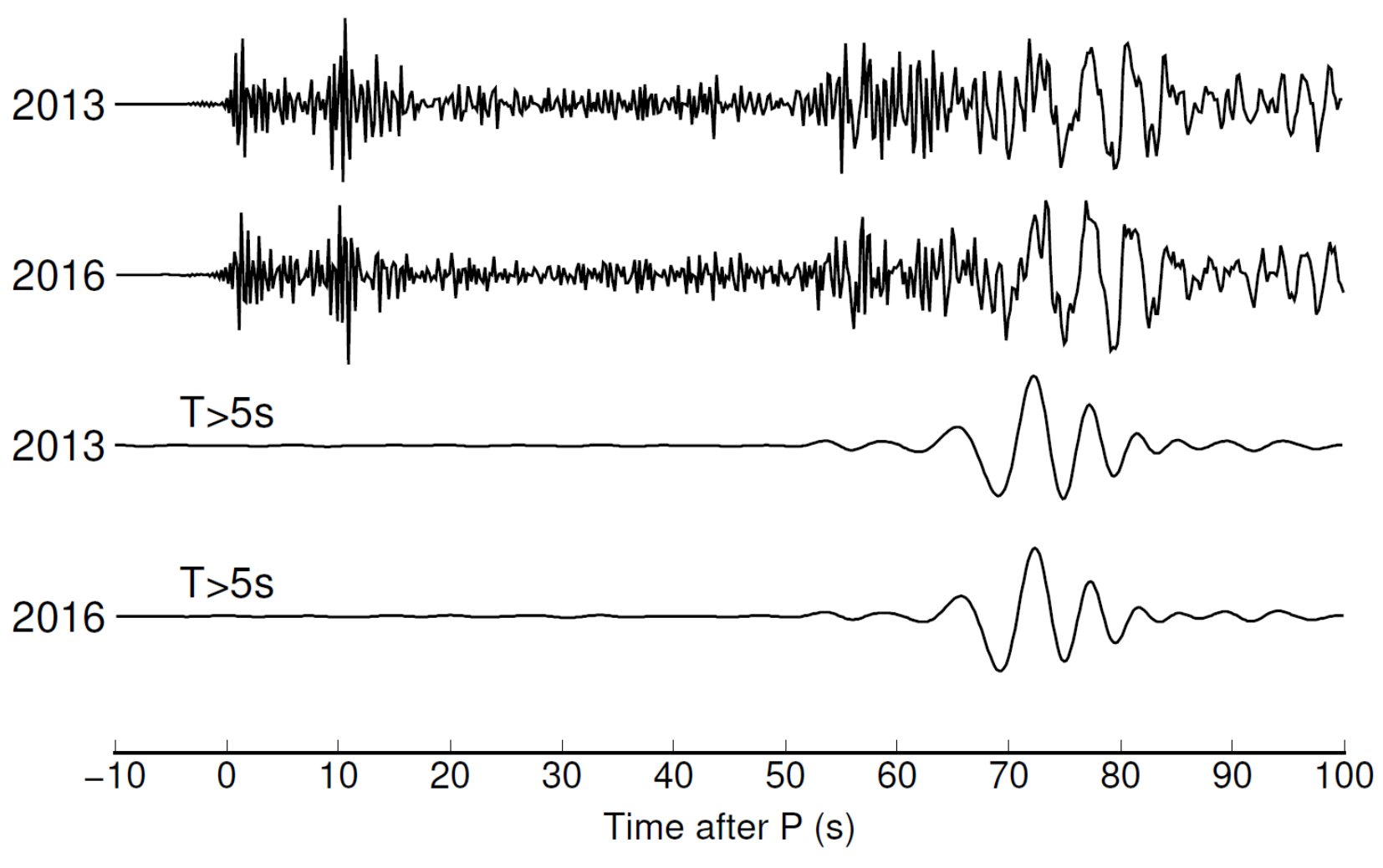

Figure 1. Waveform similarity of the Feb 2013 and Jan 2016 nuclear tests. The similarities in both broadband (top two) and low-passed filtered (bottom two) waveforms recorded at station MDJ suggest shared path/site effects. Note that $\mathrm{P}$ waves are only visible at short periods. The epicentral distance is about $400 \mathrm{~km}$. 
110 In this study, we develop a differential moment tensor inversion (diffMT) algorithm to study paired

111 seismic events in a Bayesian framework. We take amplitude ratios of various seismic phases to

112 cancel out path and site effects, and expect reduced moment tensor errors. For nuclear tests, these

113 should translate to better explosion discriminations and yield estimations. We verify the diffMT

114 algorithm using synthetic data, and apply it to three North Korea nuclear tests between 2013 and

115 2016. We compare our results with traditional waveform inversion solutions, and analyze the

116 explosion and tectonic release components of these tests.

\section{METHODS}

119 Our diffMT method refines the waveform-based moment tensor prior distribution with additional

120 differential measurements for an event pair. There are two steps. First, we apply the generalized Cut121 and-Paste (gCAP) inversion for moment tensor solutions and their uncertainties as the prior 122 information. We then measure the amplitude ratios for regional and teleseismic $\mathrm{P}$ waves, regional 123 Rayleigh and Love waves, and conduct Markov Chain Monte Carlo (MCMC) inversion on these 124 differential measurements for the posterior distributions of moment tensor components.

\subsection{Generalized Cut-and-Paste inversion for prior information}

127 Our first step is equivalent to most traditional moment tensor inversions. In this study, we use gCAP 128 (Zhu and Ben-Zion, 2013) as our main driver for the waveform inversion, as improved by Bai et al. 129 (2020) to combine near-field and teleseismic data. The CAP methodology (Zhao and Helmberger, 130 1994, Zhu and Helmberger, 1996) breaks seismograms into Pnl and S/Surface waves, and models them simultaneously but allows different time shifts between observations and synthetics to 


$$
\begin{gathered}
\Lambda^{I S O}=\zeta^{2} \\
\Lambda^{D C}=\left(1-\zeta^{2}\right) *\left(1-\chi^{2}\right) \\
\Lambda^{C L V D}=\left(1-\zeta^{2}\right) * \chi^{2} .
\end{gathered}
$$

We use the bootstrapping resampling approach (Zhan et al., 2012, Jia et al., 2017) to estimate the source parameter uncertainties, which is used as a priori constraint for the following Bayesian MCMC inversions. Calculations of Green's functions are based on the propagator matrix method with plane wave approximation (Kikuchi and Kanamori, 1991) for the teleseismic body waves, and the frequency-wavenumber integration method (Zhu and Rivera, 2002) for regional surface waves.

\subsection{Prediction and measurement of amplitude ratios}

147 We calculate amplitude ratios of regional Pn/P, teleseismic P, regional Rayleigh and Love waves

148 from two events to cancel out the path and site effects. The far-field seismic waves of an event pair can be represented by

$$
u_{1}(\boldsymbol{x}, t)=M_{i j}^{1} * G_{i j}(\boldsymbol{x}, t) * S_{1}(t) * r(\boldsymbol{x})
$$




$$
u_{2}(\boldsymbol{x}, t)=M_{i j}^{2} * G_{i j}(\boldsymbol{x}, t) * S_{2}(t) * r(\boldsymbol{x})
$$

where $M_{i j}$ is the full moment tensor, $G_{i j}$ is the Green's function, $S$ is the source time function, and $r$ is

152 the station amplification term. If we use body waves at periods longer than the source durations, we can reasonably approximate the studied events as point sources, and remove common path/site

154 effects by taking amplitude ratios. For regional and teleseismic $\mathrm{P}$ waves recorded at the same station, 155 the amplitude ratios of point sources are equivalent to their radiation pattern ratios, which is a 156 function of take-off angle and azimuth, based on ray theory being implemented in a layered elastic 157 media (Dahm, 1996).

On the other hand, the surface wave amplitude ratios are complex functions of the moment tensors and depths. When the source depth $h$ is much less than the wavelength as in the case of nuclear tests, certain surface wave eigenfunction terms are reduced to 0 ,

$$
\begin{gathered}
l_{2}(h)=\left.\mu \frac{d l_{1}}{d z}\right|_{h}=0, \\
r_{3}(h)=\left.\mu\left(\frac{d l_{1}}{d z}-k r_{2}\right)\right|_{h}=0, \\
r_{4}(h)=0,
\end{gathered}
$$

where $r$ and $l$ are components of the Rayleigh and Love wave motion-stress vectors, and the excitation of Rayleigh and Love waves is given by

$$
\begin{gathered}
\boldsymbol{u}^{R a y l}(\boldsymbol{x}, \omega)=\boldsymbol{G}^{\boldsymbol{R}}\left[U_{1}+U_{2} \cos 2 \phi+U_{3} \sin 2 \phi\right], \\
\boldsymbol{u}^{\text {Love }}(\boldsymbol{x}, \omega)=\boldsymbol{G}^{\boldsymbol{L}}\left[U_{2} \sin 2 \phi-U_{3} \cos 2 \phi\right],
\end{gathered}
$$

where $\boldsymbol{G}^{\boldsymbol{R}}$ and $\boldsymbol{G}^{\boldsymbol{L}}$ are given by 


$$
\begin{gathered}
\boldsymbol{G}^{\boldsymbol{R}}(\boldsymbol{x} ; h, \omega)=\sum_{n} \frac{k_{n} r_{1}(h)}{8 c U I_{1}} \sqrt{\frac{2}{\pi k_{n} r}} \exp \left[i\left(k_{n} r+\frac{\pi}{4}\right)\right]\left[r_{1}(z) \hat{\boldsymbol{r}}+i r_{2}(z) \hat{\mathbf{z}}\right] \\
\boldsymbol{G}^{\boldsymbol{L}}(\boldsymbol{x} ; h, \omega)=\sum_{n} \frac{i k_{n} l_{1}(h)}{8 c U I_{1}} \sqrt{\frac{2}{\pi k_{n} r}} \exp \left[i\left(k_{n} r+\frac{\pi}{4}\right)\right] l_{1}(z) \widehat{\boldsymbol{\phi}}
\end{gathered}
$$

165 in which $\mu$ is the shear modulus, $r$ is the distance, $z$ is the depth, $\hat{\boldsymbol{r}}, \hat{\mathbf{z}}, \boldsymbol{\phi}$ are the unit vectors for 3 166 cylindrical coordinates, and $k_{n}$ is the $\mathrm{n}^{\text {th }}$ root of the wave number (Aki and Richards, 2002). The radiation pattern coefficients $U_{1}, U_{2}, U_{3}$ are given by

$$
\begin{gathered}
U_{1}=\frac{1}{2}\left(\boldsymbol{M}_{x x}+\boldsymbol{M}_{y y}\right)-\left(1-\frac{2 \beta^{2}}{\alpha^{2}}\right) \boldsymbol{M}_{z z}, \\
U_{2}=\frac{1}{2}\left(\boldsymbol{M}_{x x}-\boldsymbol{M}_{y y}\right) \\
U_{3}=\boldsymbol{M}_{x y} .
\end{gathered}
$$

168 When two events E1 and E2 are both shallow and closely located, they share similar $\boldsymbol{G}^{\boldsymbol{R}}$ and $\boldsymbol{G}^{\boldsymbol{L}}$. 169 Hence these terms can be canceled out by calculating the amplitude ratios. The analytical form of 170 Rayleigh and Love wave amplitude ratios would be functions of moment tensors $\boldsymbol{M}_{E 1}, \boldsymbol{M}_{E 2}, \mathrm{Vp} / \mathrm{Vs}$ 171 ratios $\beta / \alpha$, and station azimuth $\phi$,

$$
\begin{gathered}
\left.\boldsymbol{A}^{\boldsymbol{R}}\right|_{\frac{E 1}{E 2}}=\frac{\left.\left(U_{1}+U_{2} \cos 2 \phi+U_{3} \sin 2 \phi\right)\right|_{E 1}}{\left.\left(U_{1}+U_{2} \cos 2 \phi+U_{3} \sin 2 \phi\right)\right|_{E 2}}, \\
\boldsymbol{A}^{\left.\boldsymbol{L}\right|_{\frac{E 1}{E 2}}}=\frac{\left.\left(U_{2} \sin 2 \phi-U_{3} \cos 2 \phi\right)\right|_{E 1}}{\left.\left(U_{2} \sin 2 \phi-U_{3} \cos 2 \phi\right)\right|_{E 2}}
\end{gathered}
$$

172 This means we can also take the path effects away by calculating amplitude ratios of surface waves.

174 For vertical component $\mathrm{P}$ waves, we cut 3-second time windows right after the hand-picked $\mathrm{P}$

175 arrivals, and cross-correlate to measure the amplitude ratios. We calculate two different terms,

$$
A^{1}=\frac{\int \boldsymbol{u}(\tau-t) \boldsymbol{v}(\tau) d \tau}{\int \boldsymbol{v}^{2}(\tau) d \tau}
$$




$$
A^{2}=\frac{\int \boldsymbol{u}^{2}(\tau) d \tau}{\int \boldsymbol{u}(\tau-t) \boldsymbol{v}(\tau) d \tau}
$$

176 where $\boldsymbol{u}(t)$ and $\boldsymbol{v}(t)$ are the wave segments of two events after cross-correlation. The terms $A^{1}$ and

$177 A^{2}$ are similar to the waveform-coherency-based amplitude ratio defined in an adjoint tomographic

178 inversion (Tao et al., 2017) and reflect the waveform similarity of the cross correlations. The term

$179 A^{1}$ generally represents $\boldsymbol{u} / \boldsymbol{v}$, while $A^{2}$ represents $1 /(\boldsymbol{v} / \boldsymbol{u})$ after an appropriate time shift. If $\boldsymbol{u}$ and $\boldsymbol{v}$ 180 have the same waveform shape (correlation coefficient =1), $A^{1}$ and $A^{2}$ would be identical and 181 equal to the amplitude amplification factor (AAF) (Tan and Helmberger, 2007). Otherwise, $A^{1}$ will 182 be smaller and $A^{2}$ will be larger than the AAF. Therefore, it's logical to take $A^{1}$ and $A^{2}$ as lower 183 and upper bound to assess the waveform-coherency-dependent amplitude ratio variations. We take 184 the natural logarithm of the absolute values of $A^{1}$ and $A^{2}$, and choose their mean as data and the 185 half deviation as data uncertainty. Besides, we extract the polarity difference from cross correlations confidentce limit).

Measurement of the Rayleigh and Love wave amplitude ratios and errors is similar to that of body waves. We choose the time window to be 60 s centered at the peak envelope amplitudes for cross correlations. Specifically for Rayleigh waves, we take the largest deviation between $\ln \left(\left|A^{1}\right|\right)$ and $\ln \left(\left|A^{2}\right|\right)$ for both radial and vertical components for the amplitude ratio errors.

\subsection{Bayesian Markov Chain Monte Carlo inversion}


posterior probability density functions (PDFs) by fitting the differential measurements (i.e., amplitude ratios and polarity differences) of body and surface waves. The MCMC inversion follows a Bayesian framework, which produces model distribution from data fittings and the a priori information (Tarantola, 2005),

$$
p(\boldsymbol{m} \mid \boldsymbol{d}) \propto p(\boldsymbol{m}) * l(\boldsymbol{d} \mid \boldsymbol{m})
$$

where the $p(\boldsymbol{m})$ and $p(\boldsymbol{m} \mid \boldsymbol{d})$ are prior and posterior PDFs, respectively. $d$ indicates the amplitude ratio data, including logarithmic amplitude ratios and polarity differences. $m$ represents the 6 independent source parameters $\left(M_{w}, \zeta, \chi\right.$, strike, dip, and rake) for each event, in total 12 parameters for an event pair. Conversion from the data to model is performed through the likelihood function, which describes how the predictions from a model fit the data within data error. Our likelihood function is defined as the following equation,

$$
l(\boldsymbol{d} \mid \boldsymbol{m})=\frac{1}{\sqrt{(2 \pi)^{N}\left|\boldsymbol{C}_{\boldsymbol{d}}\right|}} \exp \left(-\frac{1}{2}(G(\boldsymbol{m})-\boldsymbol{d})^{T} \boldsymbol{C}_{\boldsymbol{d}}^{-1}(G(\boldsymbol{m})-\boldsymbol{d})\right)
$$

where $G$ is the forward simulation operator, and $\boldsymbol{C}_{\boldsymbol{d}}$ is the data covariance matrix. We assume that

$\boldsymbol{C}_{\boldsymbol{d}}$ is diagonal:

$$
\begin{gathered}
\boldsymbol{C}_{\boldsymbol{d}_{i i}}=\sigma_{i}^{2}, \quad i \in[1, N] \\
\boldsymbol{C}_{\boldsymbol{d}_{(i+N)(i+N)}=\varepsilon_{i}^{2}, \quad i \in[1, N]}
\end{gathered}
$$

209 where $\sigma_{i}$ and $\varepsilon_{i}$ are the standard deviation errors of logarithmic amplitude ratio and polarity 210 difference at the $i^{\text {th }}$ station, respectively. To avoid the inversion being dominated by data points of 211 minimal errors, we set $\sigma_{i}$ to be no less than 0.05 , corresponding to $\sim 5 \%$ amplitude ratio difference. 212 Here we assumed no correlation between data errors for different stations, different phases, and 213 various measurement types (amplitude ratios vs. polarities), which may not best reflect the true 214 covariance. But because the P and surface waves are well separated, and Rayleigh and Love waves 
have orthogonal direction of vibration, their interferences are unlikely substantial. It's also reasonable to ignore the covariance between amplitude ratios and polarities, as they would be correlated only when the observation is close to the nodal, which is the minority of all stations.

We use Markov Chain Monte Carlo (MCMC) method to sample the posterior PDF $p(m \mid d)$. For low-dimension problems, brutal force algorithms are sufficient to sample the posterior PDF. When the dimensionality increases (e.g. $>10$ ), the volume of the model space increases exponentially, and the available trials become too sparse to grid-search the models. Instead, MCMC allows us to sample higher dimension distributions of known form but difficult to grid-search. Guided by the form of the posterior PDF, a Markov Chain randomly walks through the model space and results in an ensemble of models which density follows the target distribution. The models move to higher posterior probabilities with Gaussian random perturbations, and can still accept less likely models and thus jumping out of the local minimums (Hastings, 1970).

We generate 200 Markov Chains, and eventually keep 1/4 chains with highest posterior probability to avoid being trapped in low posterior minima. For each chain, we randomly generate 200 samples, and select the one of highest posterior probability as the initial draw. We apply the Gaussian proposal distributions to perturb the model at each step towards a new model. The Gaussian proposal distribution of each parameter has a standard deviation of $1 / 10$ standard deviation of its prior distribution. We follow the Metropolis Hasting algorithm (Hastings, 1970) to drive the random walk, but different from conventional Metropolis-Hasting algorithm which perturb all parameters simultaneously, we propose new models by sampling one parameter while keeping the other 
parameters at their current values (Jia et al., 2020a). The parameter being perturbed is randomly selected. This approach ensures a high acceptance rate and improves the efficiency of convergence.

Our Markov Chains usually converge in hundreds to thousands of iterations, but we choose a conservative number of burn-in samples to be 20000. After the burn-in stage, we keep the next 20000 samples in each chain, and combine 50 chains to form the final ensemble for the posterior PDFs.

\section{SYNTHETIC TEST}

We first benchmarked diffMT with synthetics, using the configuration of two collocated nuclear tests at the North Korea test site. Nuclear events have shallow burial depths and short duration, thus fitting our assumptions well. We put the pair at a depth of $0.6 \mathrm{~km}$, and with the $\mathrm{E} 1$ moment tensor as $\left(\mathrm{M}_{\mathrm{w}}\right.$ $4.53, \boldsymbol{\Lambda}^{I S \boldsymbol{O}}=86 \%$, strike $\left./ \mathrm{dip} / \mathrm{rake}=70^{\circ} / 40^{\circ} / 70^{\circ}\right)$ and the E2 moment tensor as $\left(\mathrm{M}_{\mathrm{w}} 4.44, \boldsymbol{\Lambda}^{I S \boldsymbol{O}}=73 \%\right.$, strike $/$ dip $/$ rake $=160^{\circ} / 30^{\circ} / 90^{\circ}$ ). Using these source parameters, we calculated synthetic seismograms for 8 regional (within epicentral distance of $15^{\circ}$ ) and 33 teleseismic (epicentral distance between $30^{\circ}$ and $90^{\circ}$ ) stations (Fig. 2a). The velocity model used is based on a combination of a 3-layer 1D elastic model (Ford et al., 2009) and the iasp91 model (Kennett et al., 1995). We collected real seismic noise for the used stations, and added them to the synthetic waveforms (Fig. 2b) for a similar level of signal-to-noise ratio (SNR) as natural nuclear test events. After adding the noise, the synthetic surface waves still have high SNRs, while the body waves are generally hard to observe in broadband. This is similar to the real data for most North Korea nuclear tests. 
(a)

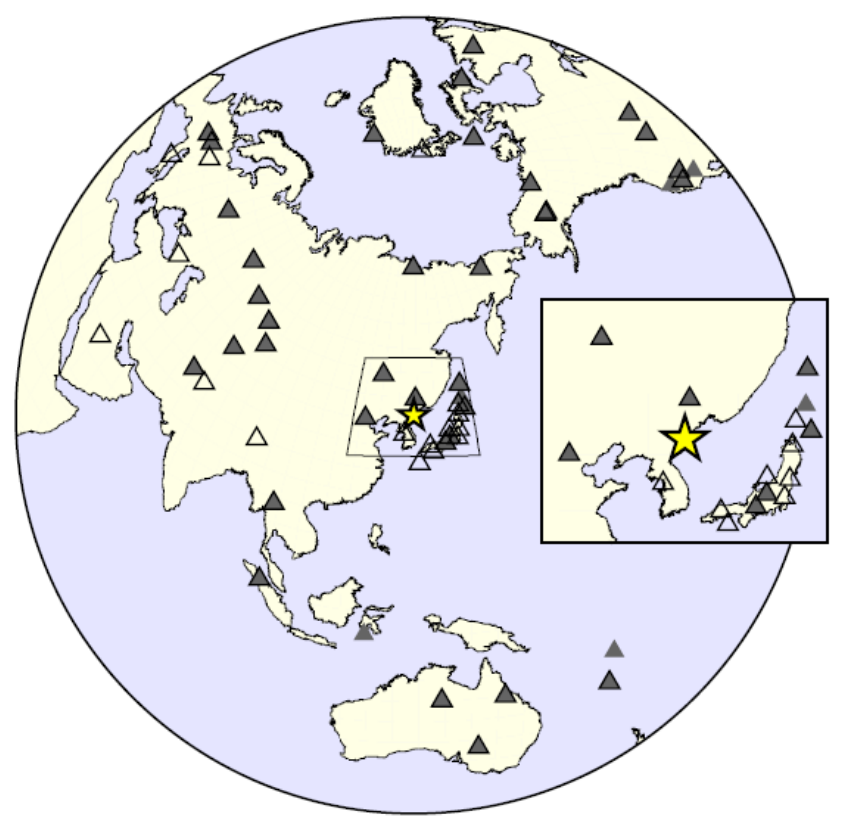

(b)

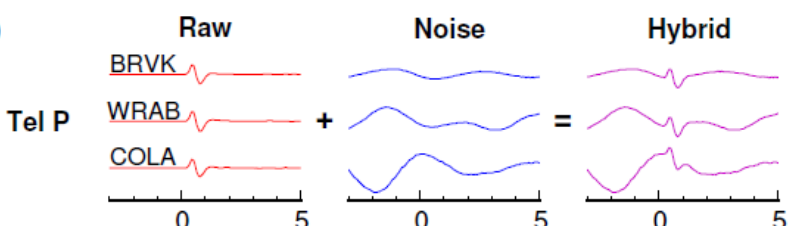

Loc $P$

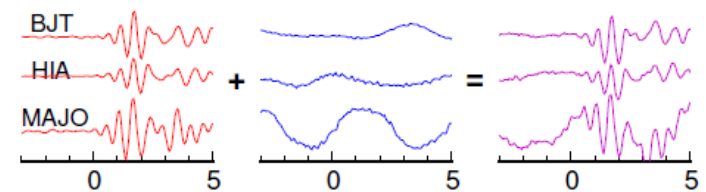

Rayl

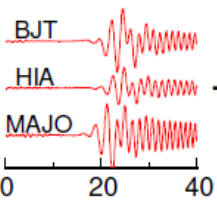

BJT

Love

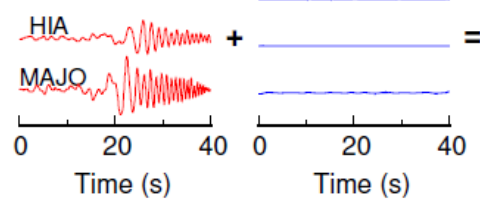

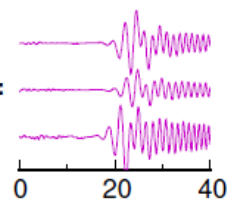

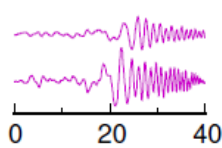

Figure 2. Generation of synthetic waveforms in our test. (A) Configuration of collocated sources (yellow star) and the seismic stations (gray triangles). Black-outlined triangles are the stations used in the following realdata inversions. The inset box shows the regional stations. (B) Adding real noise (blue lines) to the raw seismograms (red lines) for the hybrid synthetic data (purple lines).

We first applied the gCAP inversion on the two events. We filtered the data and synthetics between site amplifications of high frequency $\mathrm{P}$ waves is difficult, so we normalized the $\mathrm{P}$ waves data to the synthetic wave amplitudes and only fit the waveform shapes. We also fixed the source depths to 0.6 $\mathrm{km}$, approximated from Voytan et al. (2019), due to the limited data resolution. The moment tensor results have $\sim 60 \%$ isotropic components for both E1 and E2 (Fig. 3), which is smaller than the input model. Moreover, the double couple focal mechanisms deviate $\sim 30$ degrees from the input values. 
272 We estimated the moment tensor standard deviation errors using 200 bootstrapping resamples, and 273 observed substantial uncertainties for both E1 and E2 (Fig. S1). Given the minor data misfits (Fig. 3),

274 the nontrivial moment tensor errors reflect poor data constraints due to limited frequency band and 275 sparse network.

277 After obtaining the gCAP solutions and uncertainties, we converted them to Gaussian a priori

278 information for the diffMT inversion. We measured the amplitude ratios of regional and teleseismic $\mathrm{P}$ 279 waves, and regional Rayleigh and Love waves. We filtered the surface waves between 0.03-0.1 Hz, 280 consistent with the gCAP inversion. For the $\mathrm{P}$ waves, we applied 0.5-2.0 Hz filter band for higher 281 signal-to-noise ratios. Most waveforms of the two events show high similarity, with polarity flips for 282 some surface wave components (Fig. 4a). The amplitude ratios show clear azimuthal variations (Fig. $2834 \mathrm{~b}$ ), which are presumably caused by the radiation pattern difference of the two events. 
(a)

E1

FM $53^{\circ} / 21^{\circ} / 37^{\circ}$ Mw 4.49

ISO:61.7\% CLVD:0.3\% DC:38.0\%

Rayleigh_z Rayleigh_r Love_t
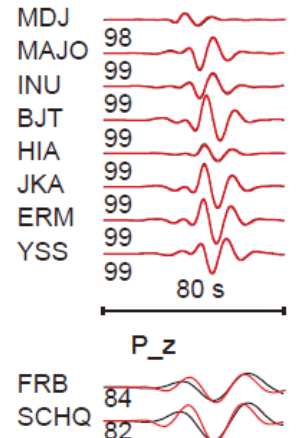

RES

BILL

INK

IL31

COLA

次

PD31 93

NV31

WDC

GSC

CMB 07

SANVU

DZM 95

NOUC $\frac{9}{6}$

CTAO $\frac{69}{99}$

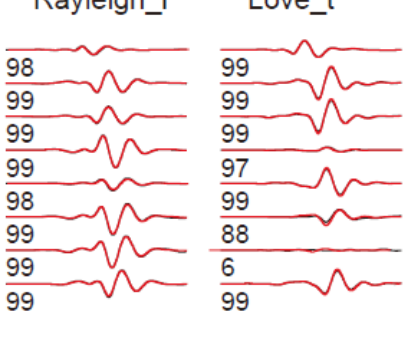

P_z

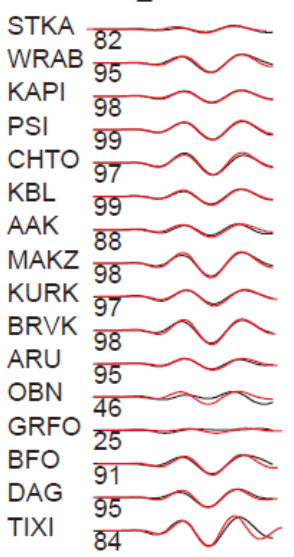

(b)

E2

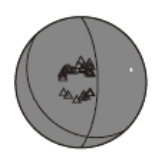

FM $137^{\circ} / 27^{\circ} / 51^{\circ}$ Mw 4.37

ISO:61.5\% CLVD:0.1\% DC: $38.4 \%$

Rayleigh_z Rayleigh_r Love_t
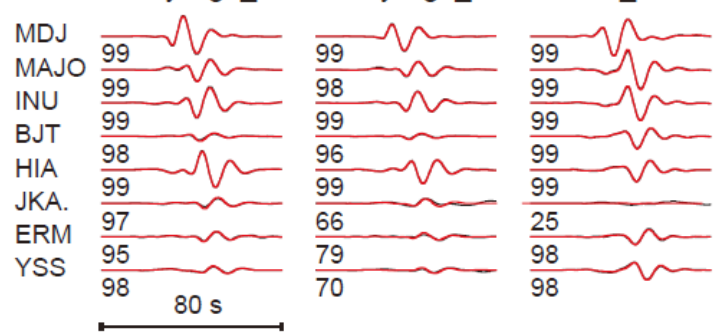

P_z

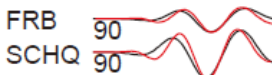

RES 81

BILL 97

INK $\overline{94}$

IL31

RSSD 83

PD31 43

NV31 27

WDC 91

GSC

CMB

SANVU

NOUC $\frac{90}{95}$

CTAO $79 \approx$

$4 \mathrm{~s}$

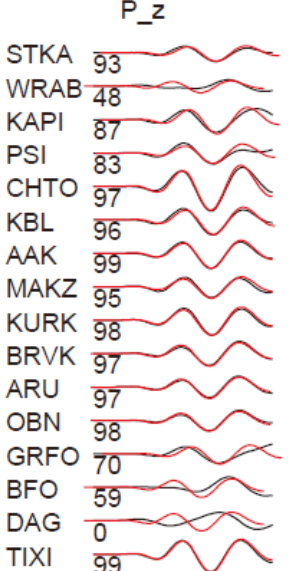

TIXI

286 Figure 3. gCAP inversion results for the two synthetic events. The black and red lines indicate data and

287 synthetic waveforms, respectively. The numbers leading the waveforms are the cross-correlation coefficients

288 between data and synthetics. 
(a)

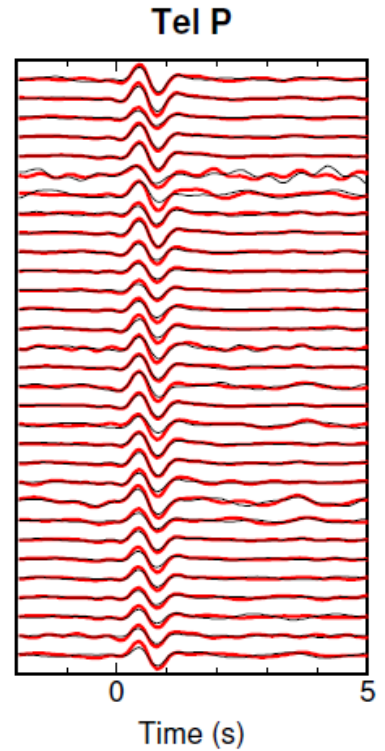

Loc P

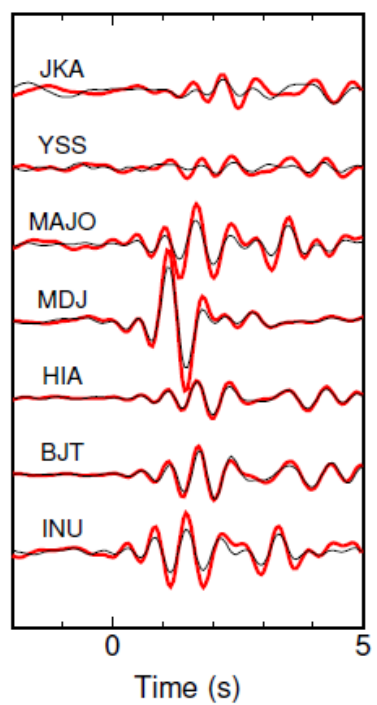

Rayl

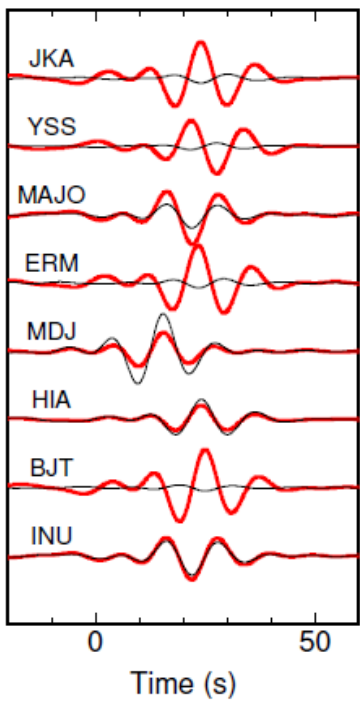

Love

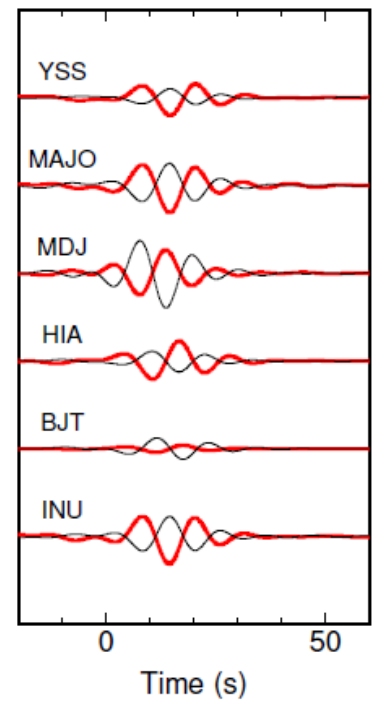

(b)

$\mathbf{P}$

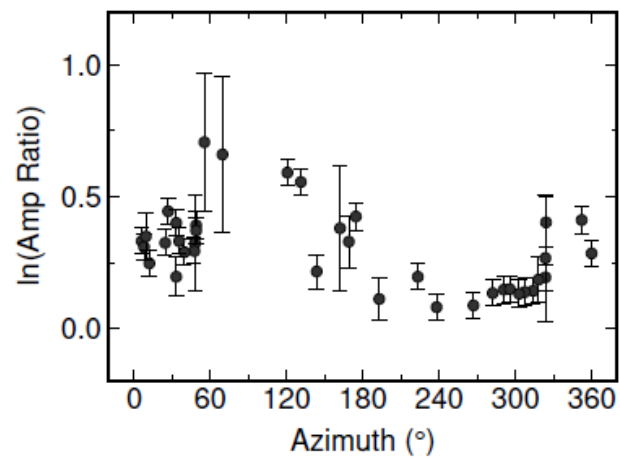

Rayl

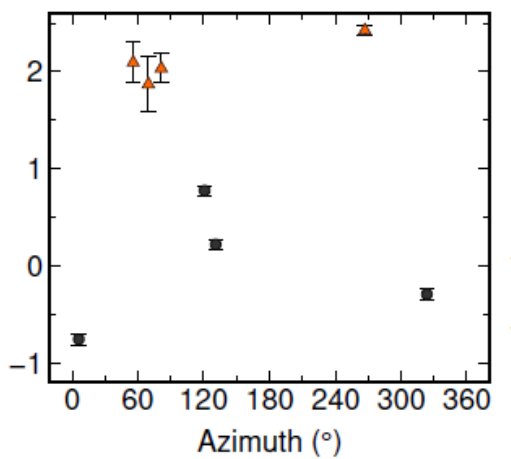

Love

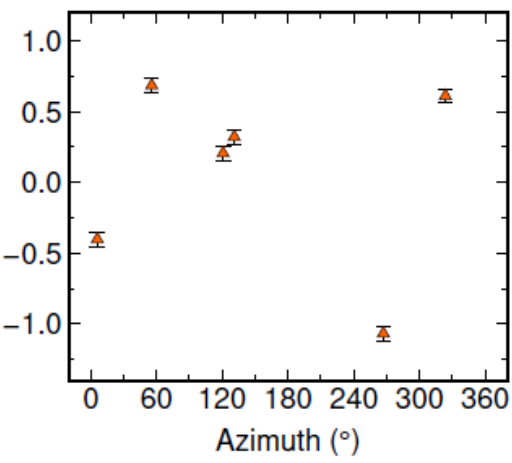

Figure 4. Measurement of amplitude ratios between two synthetic events. (A) Cross-correlated waveforms for

teleseismic P (Tel P), regional P (Loc P), Rayleigh and Love waves, respectively. (B) Amplitude ratios for P,

Rayleigh and Love waves as a function of the station azimuth. The Tel P and Loc P observations are plotted together. Black circles and orange triangles represent consistent and flipping polarities, respectively. The

With the amplitude ratio data derived from absolute amplitudes, we conducted diffMT inversion using MCMC sampling. The inversion results and data fittings are shown in Fig. 5. The posterior probability density functions (PDFs) are significantly narrower than the prior PDFs, showing reduced moment tensor uncertainties (Fig. 5a). The optimal source parameters from diffMT inversion 
are also closer to the true input values, and the moment magnitude difference is 0.08 , closer to the true difference $(0.09)$ than the prior difference $(0.12)$ (Fig. $5 b)$. The 3D rotation angle between $\operatorname{diffMT}\left(61^{\circ} / 51^{\circ} / 61^{\circ}\right)$ and true solution of E1 is $13^{\circ}$, significantly less than the $27^{\circ}$ rotation between the gCAP and true solution (Fig. 5c). Similar improvement is observed for E2, where the 3D rotation angle between diffMT $\left(150^{\circ} / 26^{\circ} / 73^{\circ}\right)$ and the true solution is $10^{\circ}$, less than the rotation angle between the gCAP and true solution $\left(22^{\circ}\right)$ (Fig. 5c). This is primarily because the azimuthal variations of the amplitude ratios, which is well fit by the diffMT synthetics (Fig. 5d), provide additional constraints that improve the moment tensor accuracy.

(a)
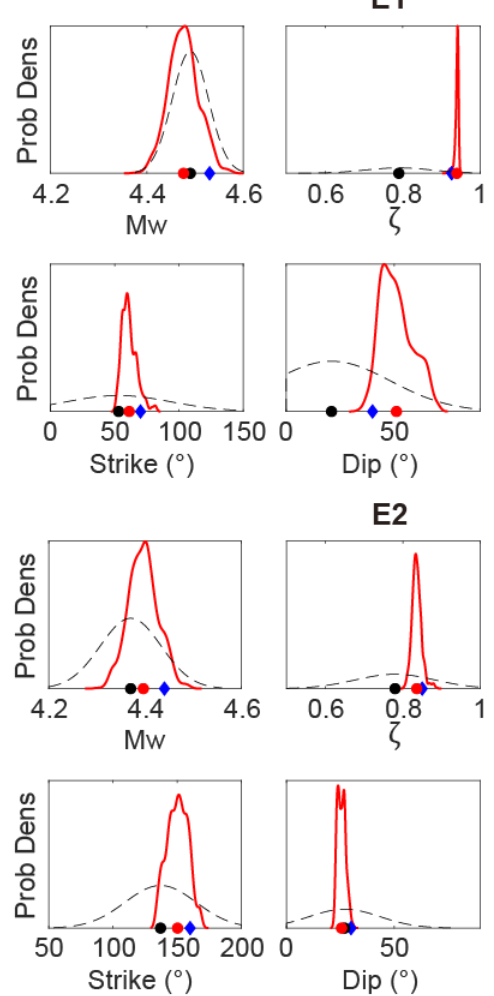

E2

E1
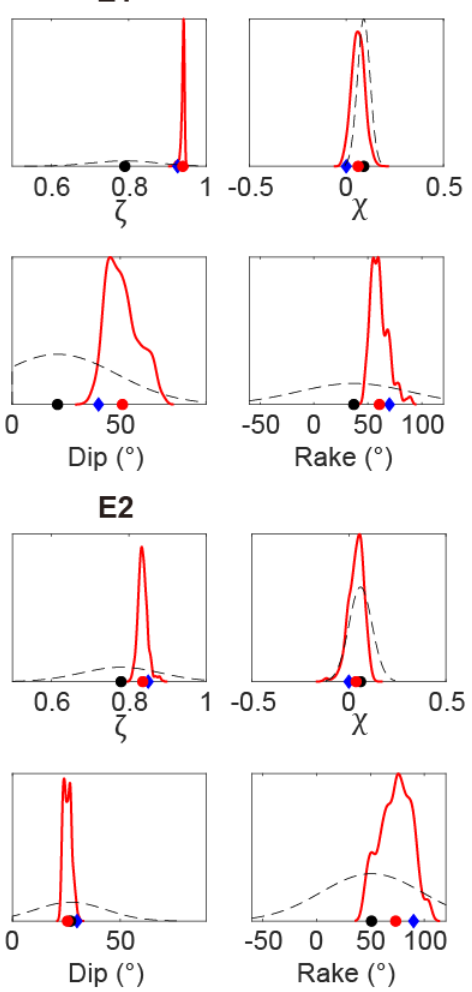

(b)

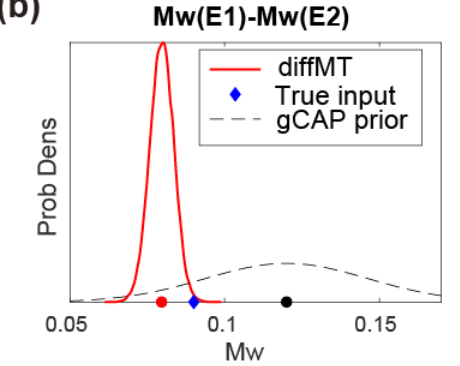

(c)

E1

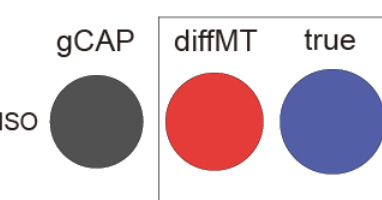

DC

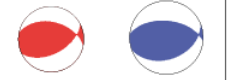

E2

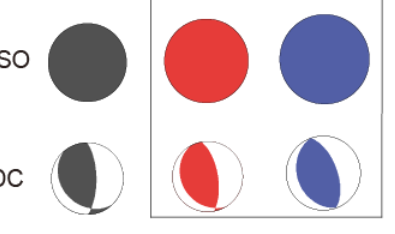

(d)
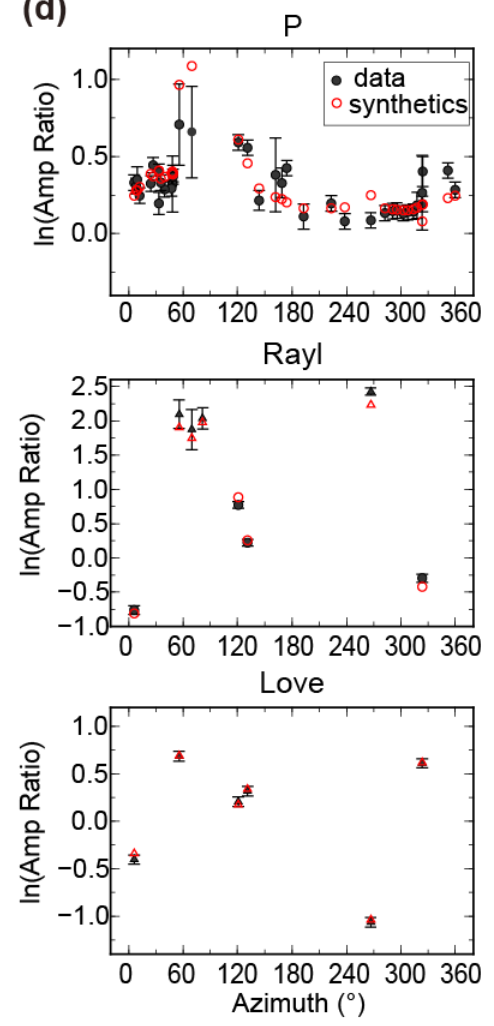

Figure 5. DiffMT inversion results for the two synthetic events. (a) The gCAP prior (dashed black lines) and the diffMT posterior (red lines) PDFs of the moment tensor solutions of the two events. The prior (gCAP) is 
from Gaussian fitting of bootstrapping uncertainties. Black and red dots indicate the gCAP optimal solution and mean of the diffMT posterior distribution, respectively. Blue diamond represents the true input value. See legend in (b). (b) The prior (dashed black lines) and posterior (red lines) PDFs of the moment magnitude difference between E1 and E2. Symbols are similar to that in (a). (c) Comparison of the isotropic (DC) and double couple (DC) focal mechanisms for the gCAP (black) and diffMT (red) solutions. Blue beachballs show the true focal mechanisms. The sizes of beachballs are proportional to the corresponding magnitudes. (d) Amplitude ratio fittings for the diffMT solution. Black squares and red symbols show the amplitude ratio data and predictions from the moment tensor models, respectively. Circles and triangles represent consistent and flipping polarities, respectively.

\section{APPLICATION ON NORTH KOREA NUCLEAR TESTS}

We applied our diffMT algorithm to the three North Korea nuclear tests on Feb 2013, Jan 2016 and

Sep 2016, respectively, by conducting inversions on three event pairs using seismograms from regional (within epicentral distance of $15^{\circ}$ ) and teleseismic (epicentral distance between $30^{\circ}$ and $90^{\circ}$ ) stations (Fig. 3). The number of observations for all 3 events are not identical due to the varying station availability across the time period, but since the overlapping stations are the majority, the azimuthal and distance coverage differences are trivial. Similar to the synthetic test, we first run gCAP inversion using the regional surface waves in velocity filtered between $0.03-0.1 \mathrm{~Hz}$, and the teleseismic $\mathrm{P}$ waves in velocity filtered between $0.5-1.0 \mathrm{~Hz}$. The narrow $\mathrm{P}$ wave filter band is a compromise between signal observability and modeling capability. We fixed the depths to be $0.6 \mathrm{~km}$, similar to the estimations from Voytan et al. (2019), to avoid depth ambiguities. The inversion results show that both the regional and teleseismic waveforms are fit well (Fig. 6). We observe 50 70\% 
337 isotropic component for these events, which is generally consistent with other moment tensor

338 inversion studies (Cesca et al., 2017, Chiang et al., 2018, Ford et al., 2009). The distributions of the

339 moment tensors estimated from the bootstrapping resampling suggest that all the source parameter

340 components have large uncertainties (Fig. S2). Particularly, the isotropic component fraction and

341 double couple orientations are not well constrained. The wide range of model uncertainties makes it

342 difficult to discriminate the explosions or to analyze the tectonic release mechanisms.

(a)

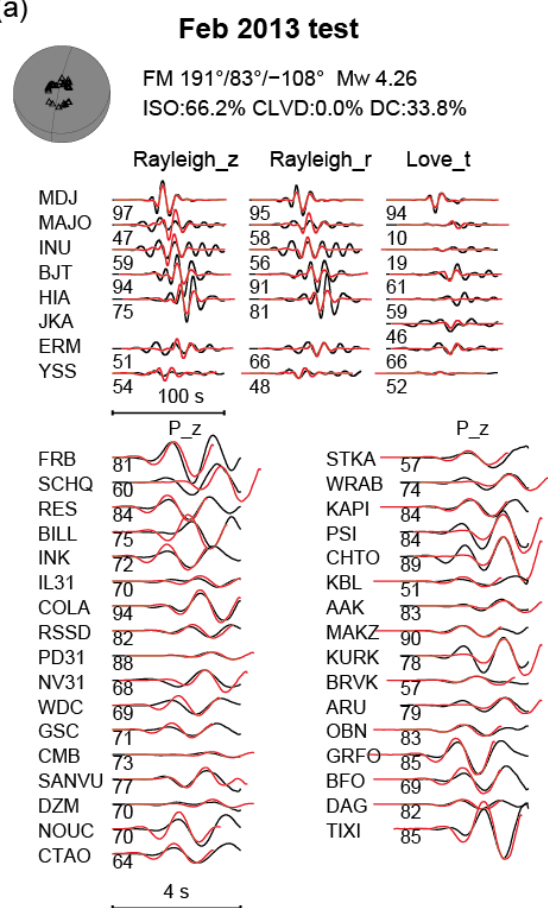

(b)

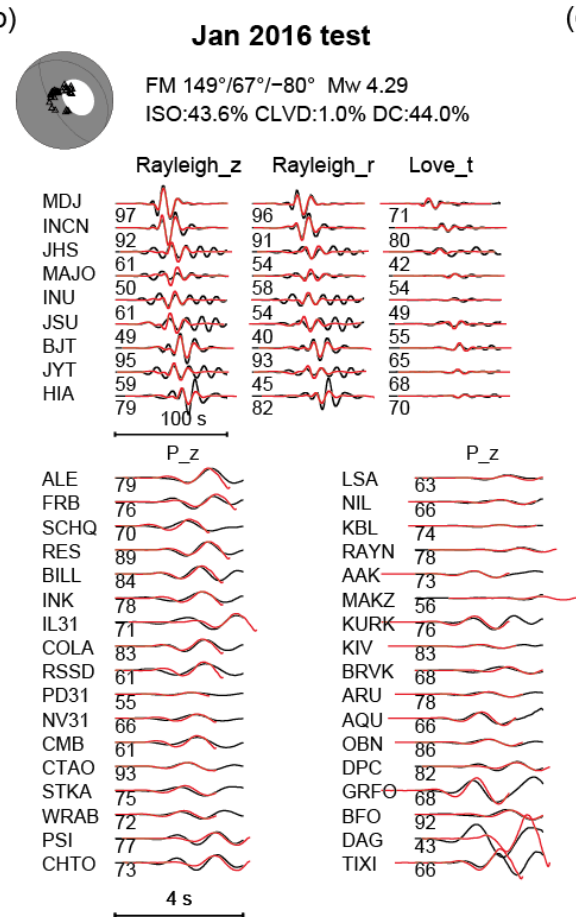

(c)

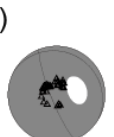

Sep 2016 test

FM $158^{\circ} / 80^{\circ} /-82^{\circ}$ Mw 4.49

ISO:52.4\% CLVD:0.1\% DC: $47.5 \%$

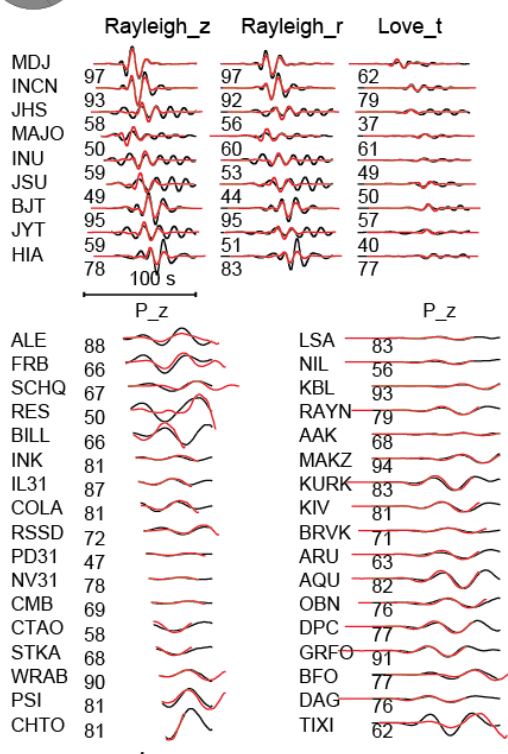

$4 \mathrm{~s}$

345 Figure 6. gCAP inversion results for the 3 studied North Korea nuclear explosions on (A) Feb 2013, (B) Jan

3462016 and (C) Sep 2016, respectively. The symbols are similar to that in Fig. 3.

348 We measure amplitude ratios of the 3 event pairs among these three tests, using regional and

349 teleseismic P waves between $0.5-2.0 \mathrm{~Hz}$, and the Rayleigh and Love waves between 0.03-0.1 Hz.

350 Waveforms of different events show high similarity, indicating robust measurements of the amplitude 
(a)
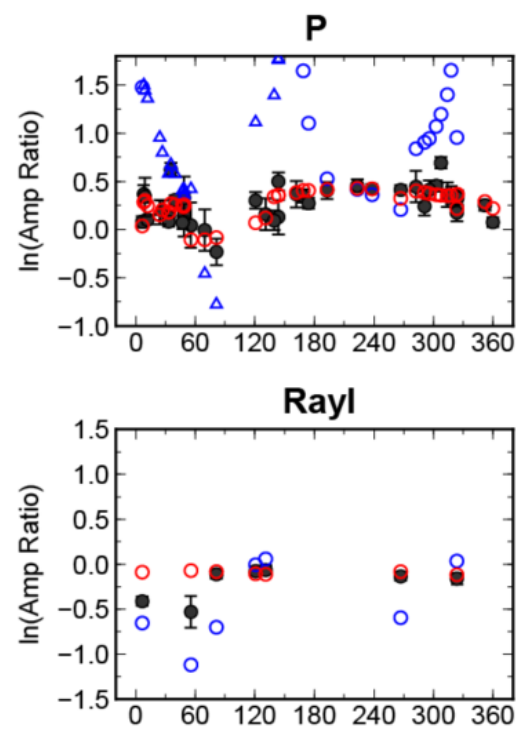

Love

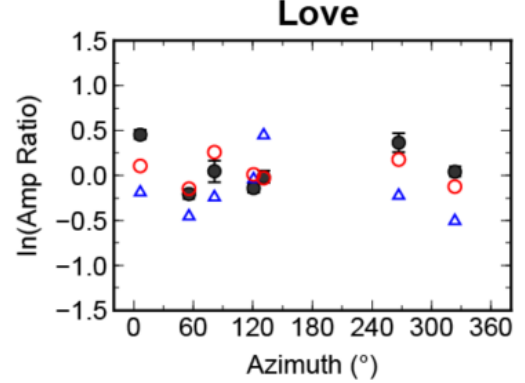

(b)
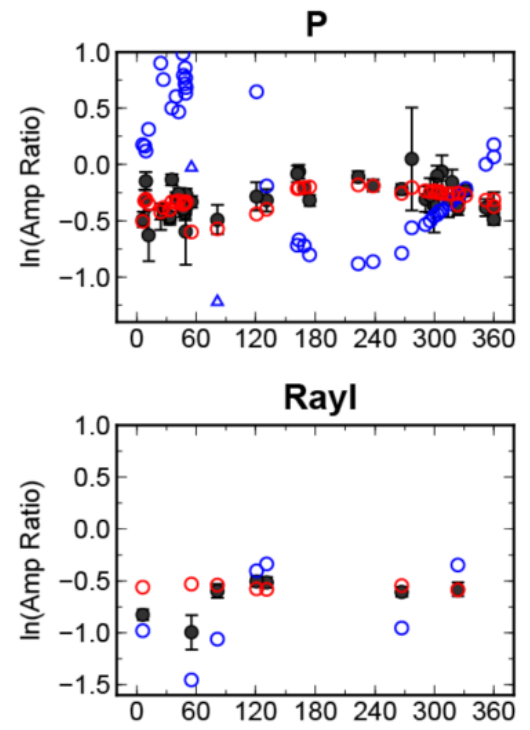

Love

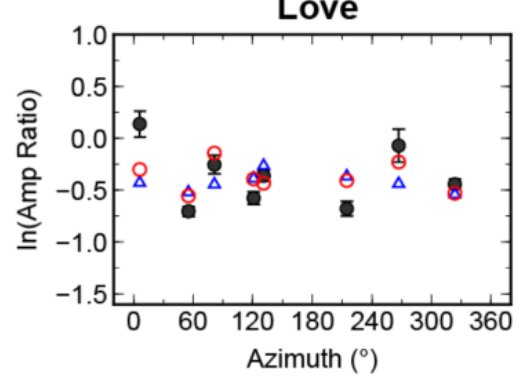

(c) 2016a-2016b

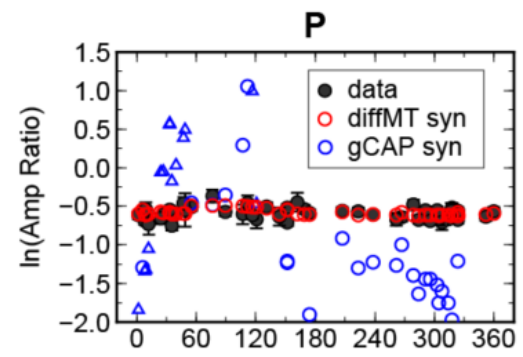

Rayl

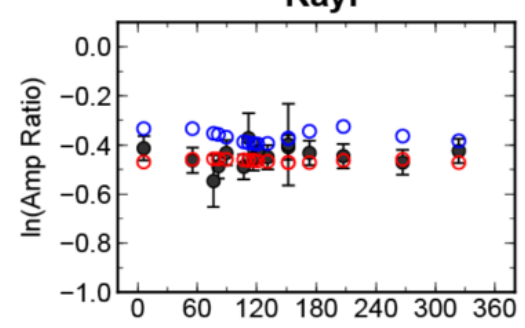

Love

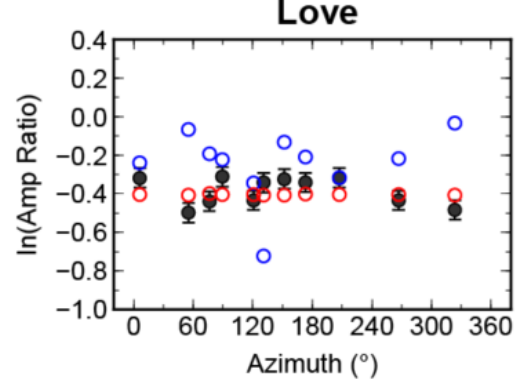

Figure 7. Amplitude ratios among the Feb 2013, Jan 2016 (2016a), and Sep 2016 (2016b) events. Three pairs are shown in (a)-(c), respectively. Circles and triangles represent consistent and flipping polarities,

respectively. Black symbols with error bars show the amplitude ratio measurements. Blue and red symbols indicate the amplitude ratio predictions from the gCAP and diffMT solutions, respectively.

We further applied three separate diffMT inversions on these pairs. We did not choose doing one 
inversion for all events, due to the rapidly growing number of unknowns $\left(\mathrm{N}^{*} 6\right)$ for $\mathrm{N}$ events, which would pose a significant challenge to the nonlinear searching efficiency. Conducting multiple paired inversions would be the most applicable way of diffMT application on the real-world seismic event clusters. To avoid the inversion being trapped to pure isotropic sources $(\zeta=1)$ which generates very low Love wave amplitudes and numerically unstable ratios, we tapered the prior of $\zeta$ (equation 1) from its maximum bootstrapping value (0.98/0.96/0.96 for the Feb 2013, Jan 2016, and Sep 2016 events) to 1 . The existence of Love waves also does not support pure isotropic source mechanisms. The diffMT posterior probability density functions (PDFs) are shown in Fig. 8. The posterior PDFs for each event are generally consistent from different pairs (Fig. 8a). Still, we can observe mismatches for some components, such as the rake angle for the Feb 2013 event, and CLVD parameter for the two 2016 tests (Fig. 8a). This is because models that fit amplitude ratio data for different pairs can have different biases from varying data errors. As long as they have overlapping 374 model space, they do not contradict each other since the overlapped models could fit the data for 375 both pairs. On the other hand, the CLVD factor $\chi$ may not be well constrained, because the CLVD 376 component is a minor term accompanied with the DC mechanism (Zhu and Ben Zion, 2013), while 377 the DC part is already second order compared to the dominant isotropic mechanism. 
(a)
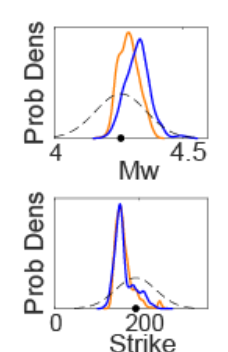

Feb 2013
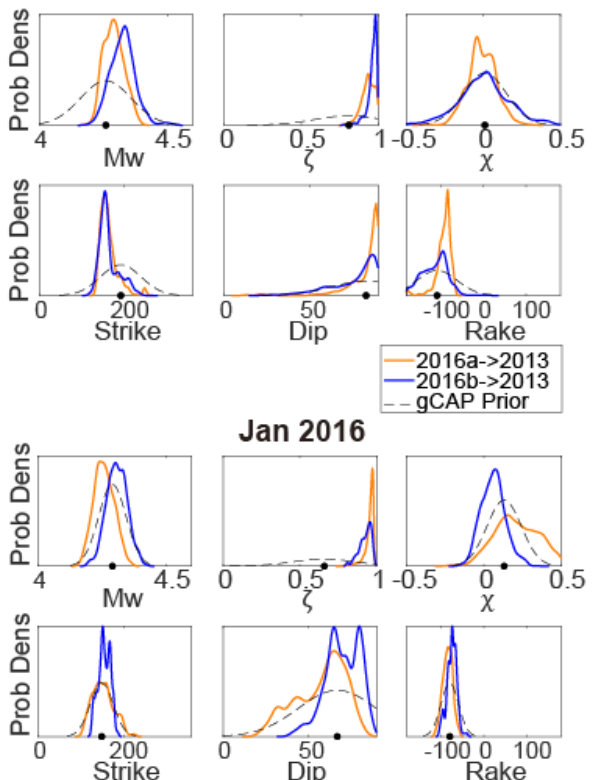

Jan 2016
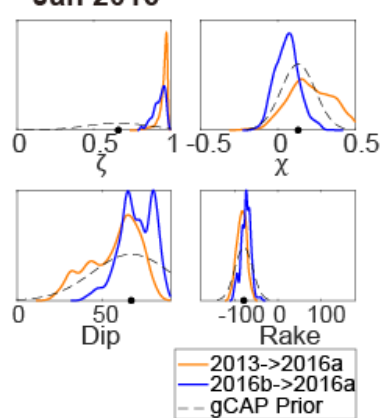

Sep 2016
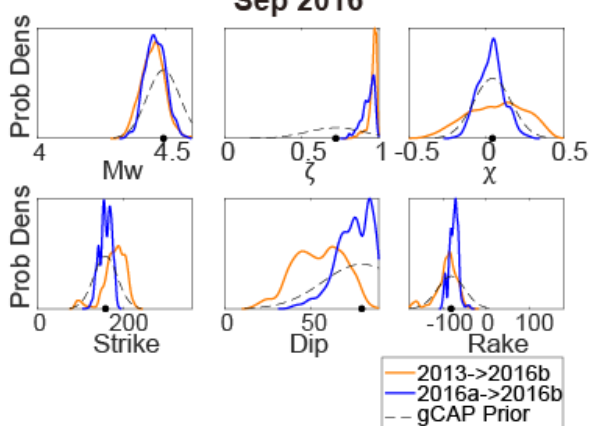

(b)
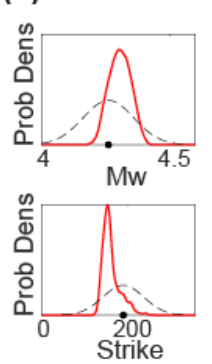

Feb 2013
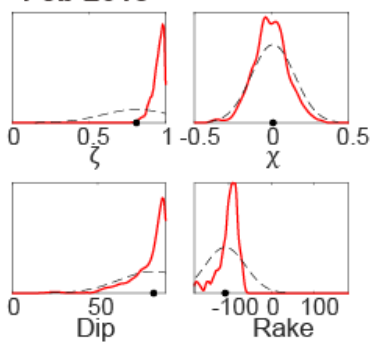

Jan 2016
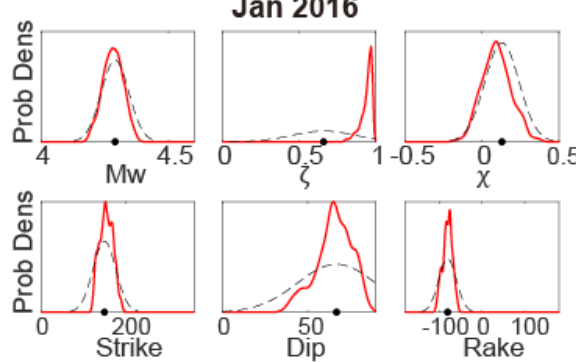

Sep 2016
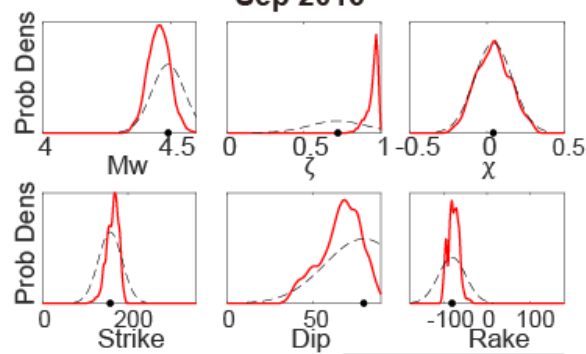

(c)

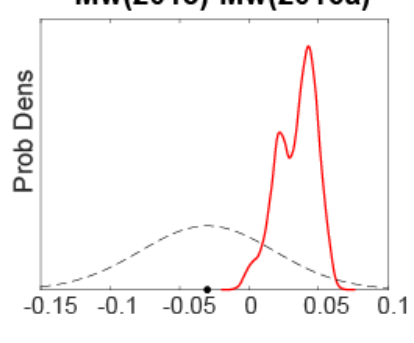

$M w(2013)-M w(2016 b)$

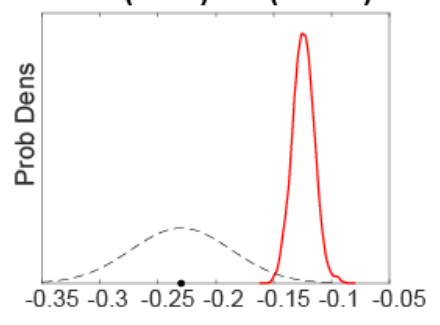

$M w(2016 a)-M w(2016 b)$
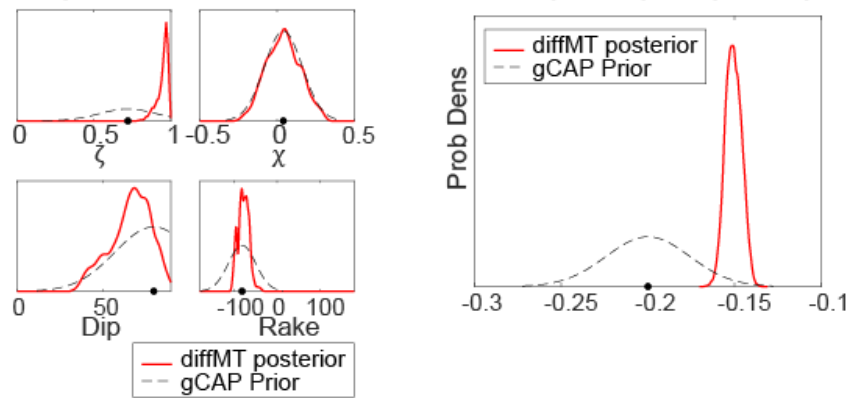

Figure 8. DiffMT inversion results for the event pair of the Feb 2013 and Jan 2016 tests. (a) The gCAP prior

381 (dashed black lines) and the diffMT posterior (solid lines) PDFs of the moment tensor solutions of the two

events. Solid lines in different colors indicate the posterior PDFs derived with different pairing events. Black

dots show the optimal gCAP solution. (b) Combined posterior PDFs (solid red lines) plotted with the gCAP

prior PDFs (dashed black lines). (c) The prior (dashed black lines) and posterior (red lines) PDFs of the

moment magnitude differences. 
Feb 2013

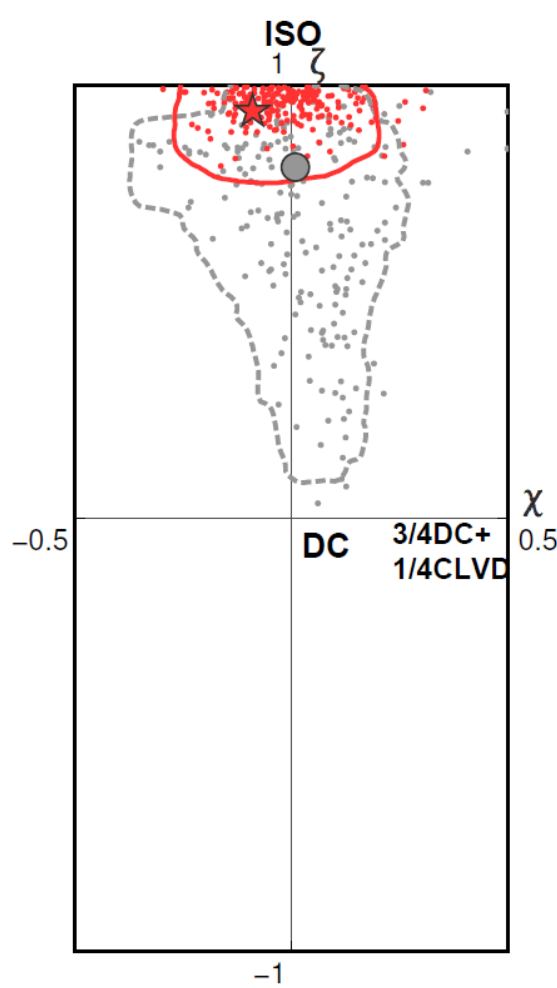

Jan 2016

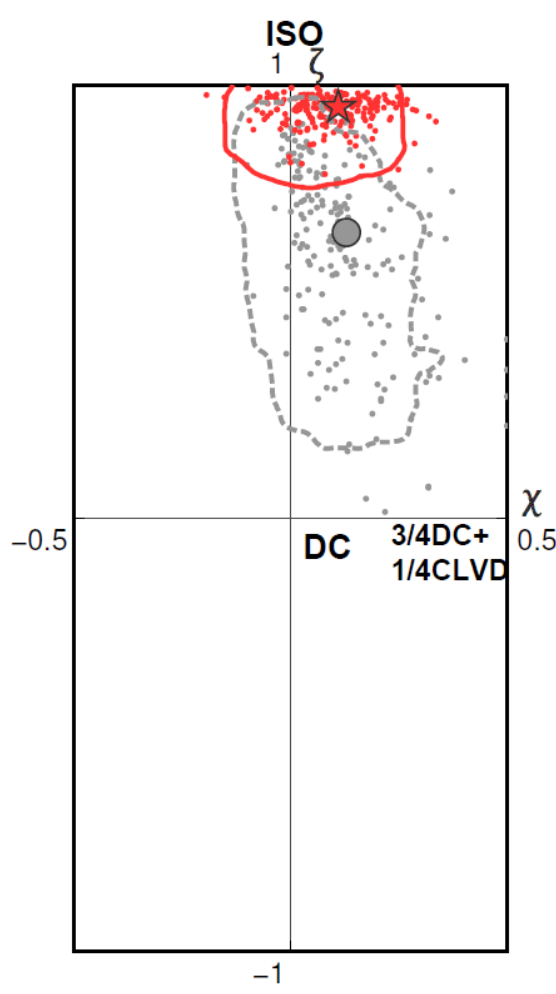

Sep 2016

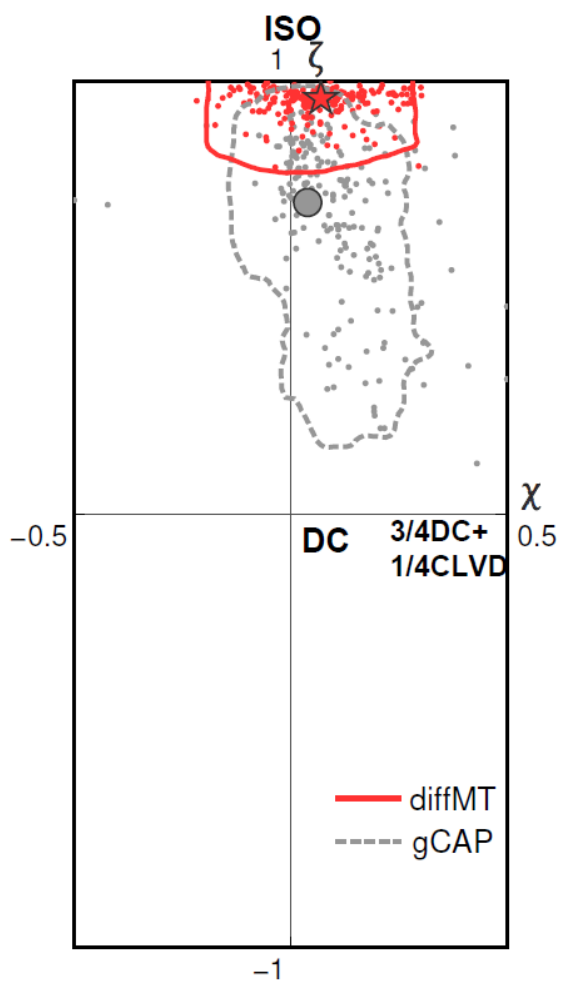

Figure 9. Prior (gCAP) and posterior (diffMT) distributions of $\zeta$ and $\chi$ for the three studied events. Red star and gray circle indicate diffMT and gCAP solution, respectively. The diffMT samples (red scattered dots) are contoured by the $90 \%$ confidence limit lines (red solid line), while the gray scattered dots and dashed lines are the gCAP samples and $90 \%$ confidence limits.

We multiplied the diffMT posterior PDFs of each event from different pairs for the overall posterior distributions (Fig. 8b). The posterior PDFs are significantly narrower than the prior PDFs, suggesting tighter constraints from the amplitude ratio measurements. In particular, the proportion of the isotropic components $\left(\zeta^{2}\right)$ are much better resolved and significantly more dominant $(\sim 90 \%)$ than the prior distributions (Fig. 9), which strongly suggest explosive source mechanisms. Meanwhile, diffMT inversions reduce the uncertainties of the moment magnitude differences (average standard deviation error of 0.04 for prior and 0.01 for posterior) (Fig. $8 \mathrm{c}$ ), and make it much easier to compare 
the size of these nuclear tests. Therefore, the diffMT results could improve explosion discrimination and size comparison for the studied North Korea nuclear tests.

Moreover, diffMT inversion significantly reduces the uncertainty of the DC component (strike, dip, rake in Fig. 8b). To illustrate the improvement, we compared the double couple focal mechanism ensembles for the gCAP prior and diffMT posterior distributions (Fig. 10). The gCAP prior ensemble similar high angle dip-slip as the tectonic release for the three nuclear tests.

(a)

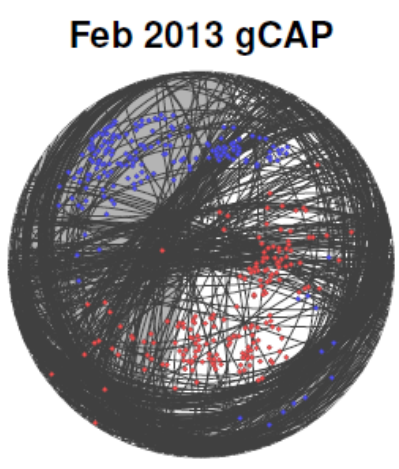

(b)

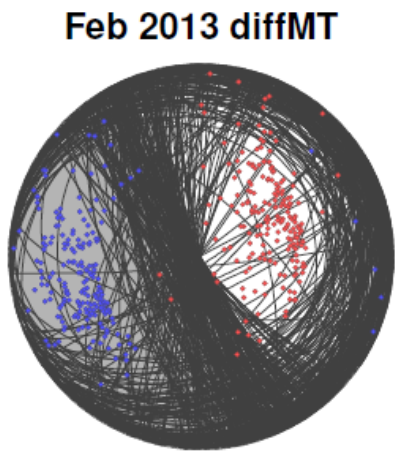

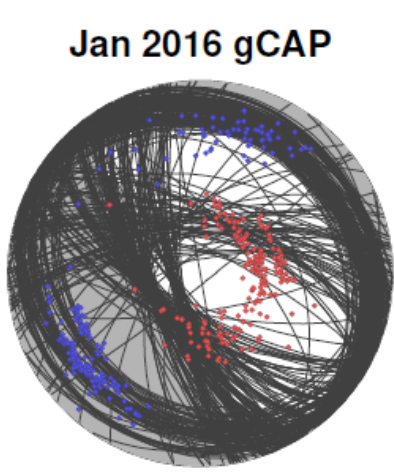

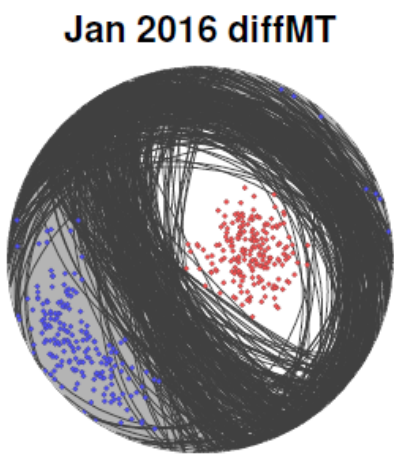

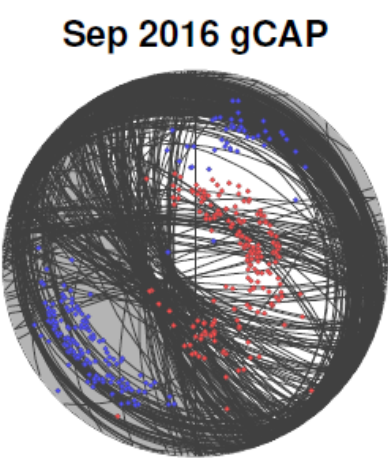

Sep 2016 diffMT

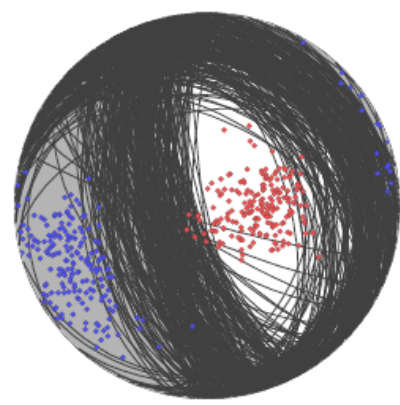

410 Figure 10. Scatter plot of the focal mechanisms from the (a) gCAP prior and (b) diffMT posterior ensembles.

411 Red and blue dots are the $\mathrm{P}$ and $\mathrm{T}$ axes of the focal mechanisms, respectively. 


\section{DISCUSSION}

414 Our application of the diffMT inversion on the North Korea nuclear tests shows better-resolved moment tensors. Although the gCAP inversion uses absolute body and surface wave amplitudes, it does not capture the patterns of amplitude ratios which provide extra constraints on moment tensors. The gCAP and diffMT solutions fit the regional and teleseismic waveforms almost equally well (Fig. S4), suggesting that the absolute amplitude information can hardly distinguish the two moment tensor solutions. In contrast, our final diffMT solution, which is sampled near the mean of the posterior distributions, fits amplitude ratios significantly better than the gCAP solution (Fig. 7). This is because the absolute amplitude information contains the unknown path and site effects that cause misfits that translates to model uncertainties assuming a simple velocity model. On the other hand, diffMT does not require highly accurate velocity models, thus finding better MT solutions from the gCAP ensembles. methods, our diffMT inversion uses a two-step approach to combine the waveforms with the amplitude ratio information, and quantify moment tensor uncertainties in a Bayesian framework, which provides a natural uncertainty analysis. Introducing the Bayesian framework also eliminates the need of choosing reference events, and avoids the magnitude trade-offs with constraints from the priors. Moreover, diffMT includes surface waves, making it suitable for events with sparse local observations. However, our method still introduces certain assumptions which may bring additional model errors. We did not consider depth phases in the $\mathrm{P}$ wave amplitude ratio modeling. Although 
the influence of 1D depth phases appears insignificant (Fig. S5), the impact of depth phase variations due to the 3D surface topographic reflections are moderate and need further investigations(Avants, 2014, Rodgers et al., 2010). Besides, the calculations of body wave amplitude ratios rely on take-off angles calculated with a layered model, and the influence of the source side structural heterogeneities on the ray parameters is presumably low but not negligible. Overall, structural heterogeneities may still bias our inversion results, suggesting that full numerical wavefield simulations with more realistic earth models are needed in the future.

We summarized our final diffMT solutions in Table 1. The proportions of the isotropic components are all around 90\% (Table 1), substantially more dominant than the gCAP estimates of around 50$70 \%$, or some other solutions of $50 \%-60 \%$ for the North Korea nuclear tests (Vavryčuk and Kim, 2014, Cesca et al., 2017, Ford et al., 2009). Note that surface waves alone can not discriminate the isotropic and vertical-dipping CLVD sources, as their radiation patterns are similar around the edge of focal sphere. However, the P waves can cover the central portion of the beachball, and the strength of the azimuthal-varying $\mathrm{P}$ amplitude ratios constrains how much they deviate from uniform radiation (isotropic source). The moment magnitudes of these three events are 4.31, 4.28, 4.43, respectively, suggesting similar sizes for the Feb 2013 and Jan 2016 tests, followed by the larger Sep 2016 test. Double couple components are mostly dip-slip normal faulting events, and the steep dip angles of tectonic release are suggested by various studies in this region (Cesca et al., 2017, Ford et al., 2009, Barth, 2014). The DC orientations are also consistent with Cesca et al. (2017). But similar to the bottlenecks of most moment tensor inversion, our diffMT algorithm only resolve point source moment tensors for events with clear body and surface waves. Therefore, we skipped the 2009 North 
457 Korea nuclear test in our study due to the low SNRs (Fig. S6). We also did not include the Sep 2017 458 test (M 6.3), because it likely involves sequential explosions, tectonic releases and collapses (Xu et 459 al., 2020), which introduces wave complexities (Fig. S6) beyond the point-source assumption.

460 Further investigations of time-dependent source parameters are needed for large and complicated 461 nuclear explosions.

462 The ISO/DC/CLVD decomposition used in this paper, while used extensively in nuclear monitoring 463 (Ford et al., 2009, Cesca et al., 2017, Vavryčuk and Kim, 2014, Chiang et al., 2018), is not the only physical interpretation. Following Aki\&Richard's classical model, full moment tensor could be viewed as oblique opening of the fault for one of the two non-perpendicular planes (Aki and Richards, 2002, Tape and Tape, 2013). Also, a moment tensor can be decomposed as a crack tensor plus a double couple (CDC), in which the tensile crack direction is perpendicular to the fault plane of shear motion (Tape and Tape, 2013, Alvizuri and Tape, 2018). These various kinematic expressions of seismic source can lead to different understandings of the physical processes of nuclear tests. encounter wrap-around at the boundaries. In practice, we truncated the Gaussian functions at the boundaries to avoid jumps for strike/dip/rake. Although moderate changes of prior shape won't significantly influence the diffMT inversion, there are better ways to avoid the non-uniformity of the tensors. This way leads to even distributions of moment tensors in the parameter space, which could benefit the prior selection for the diffMT in the future. 
Table 1. Moment tensor solutions for the 3 studied North Korea nuclear tests.

481

\begin{tabular}{|c|c|c|c|c|c|c|c|c|c|}
\hline & $\mathbf{M}_{\mathbf{w}}$ & $\zeta$ & $\chi$ & Strike & Dip & Rake & $\Lambda^{I S O}(\%)$ & $\Lambda^{D C}(\%)$ & $\Lambda^{C L V D}(\%)$ \\
\hline Feb 2013 (prior) & $4.26_{-0.20}^{+0.20}$ & $0.81_{-0.44}^{+0.19}$ & $0.01_{-0.14}^{+0.14}$ & $191_{-96}^{+96}$ & $83_{-40}^{+7}$ & $-108_{-72}^{+94}$ & $66_{-50}^{+34}$ & $34_{-34}^{+50}$ & $0_{-0}^{+2}$ \\
\hline Feb 2013 (diffMT) & $4.31_{-0.07}^{+0.06}$ & $0.94_{-0.07}^{+0.06}$ & $-0.09_{-0.1}^{+0.27} \mid$ & $154_{-17}^{+51}$ & $79_{-22}^{+10}$ & $-92_{-58}^{+21}$ & $88_{-12}^{+12}$ & $12_{-11}^{+11}$ & $\mathbf{0}_{-0}^{+1}$ \\
\hline Jan 2016 (prior) & $4.29_{-0.10}^{+0.10}$ & $0.66_{-0.40}^{+0.34}$ & $0.13_{-0.22}^{+0.22}$ & $149_{-53}^{+53}$ & $67_{-46}^{+23}$ & $-80_{-41}^{+41}$ & $44_{-37}^{+56}$ & $56_{-43}^{+27}$ & $0_{-0}^{+8}$ \\
\hline Jan 2016 (diffMT) & $4.28_{-0.07}^{+0.07}$ & $0.95_{-0.09}^{+0.03}$ & $0.11_{-0.17}^{+0.15}$ & $161_{-33}^{+18}$ & $61_{-16}^{+20}$ & $-90_{-10}^{+28}$ & $91_{-17}^{+5}$ & $9_{-6}^{+17}$ & $\mathbf{0}_{-0}^{+1}$ \\
\hline Sep 2016 (prior) & $4.49_{-0.13}^{+0.13}$ & $0.72_{-0.37}^{+0.28}$ & $0.04_{-0.24}^{+0.24}$ & $158_{-56}^{+56}$ & $80_{-46}^{+10}$ & $-82_{-64}^{+64}$ & $52_{-40}^{+48}$ & $48_{-42}^{+40}$ & $0_{-0}^{+6}$ \\
\hline Sep 2016 (diffMT) & $4.43_{-0.05}^{+0.09}$ & $0.96_{-0.09}^{+0.03}$ & $0.07_{-0.21}^{+0.16}$ & $163_{-25}^{+21}$ & $66_{-23}^{+18}$ & $-89_{-10}^{+30}$ & $92_{-16}^{+6}$ & $8_{-6}^{+16}$ & $0_{-0}^{+1}$ \\
\hline
\end{tabular}

\section{CONCLUSIONS}

485 We developed a differential moment tensor (DiffMT) inversion algorithm that resolves moment tensors of clustered seismic event pairs using relative measurements. It starts with a conventional moment tensor inversion for the a priori solutions, followed by inversion on amplitude ratio information for the refinements. Application of diffMT on three North Korea nuclear tests between 2013 and 2016 leads to reduced errors of isotropic components and double couple focal mechanisms.

Their moment tensors have $\sim 90 \%$ explosive components, which are more dominant compared with some conventional results of $50 \% \sim 60 \%$, providing opportunity for better explosion discrimination. 
492 The associated tectonic release components are small but nontrivial high angle dip-slip mechanisms.

493 The seismic moment differences between events are also better resolved, which could improve

494 energy estimation of nuclear tests. With tighter constraints on the double couple focal mechanisms,

495 we expect the diffMT method to be applied to various types of seismic events. 


\section{REFERENCES}

Aki, K. \& Richards, P.G., 2002. Quantitative seismology, edn, Vol., pp. Pages.

Alvizuri, C. \& Tape, C., 2018. Full moment tensor analysis of nuclear explosions in North Korea, Seismol. Res. Lett., 89, 2139-2151.

Avants, M., 2014. Effects of near-source heterogeneity on wave fields emanating from crustal sources observed at regional and teleseismic distances, UC Santa Cruz.

Bai, Q., Ni, S., Chu, R. \& Jia, Z., 2020. gCAPjoint, A Software Package for Full Moment Tensor Inversion of Moderately Strong Earthquakes with Local and Teleseismic Waveforms, Seismological Society of America, 91, 3550-3562.

Ballard, S., Hipp, J.R., Begnaud, M.L., Young, C.J., Encarnacao, A.V., Chael, E.P. \& Phillips, W.S., 2016. SALSA3D: A tomographic model of compressional wave slowness in the Earth's mantle for improved travel-time prediction and travel-time prediction uncertainty, Bull. Seismol. Soc. Am., 106, 2900-2916.

Barth, A., 2014. Significant release of shear energy of the North Korean nuclear test on February 12, 2013, JSeis, 18, 605-615.

Bazargani, F., Hale, D. \& Hayes, G.P., 2013. Tensor-Guided Fitting of Subducting Slab Depths, Bull. Seismol. Soc. Am., 103, 2657-2669.

Bozdağ, E., Peter, D., Lefebvre, M., Komatitsch, D., Tromp, J., Hill, J., Podhorszki, N. \& Pugmire, D., 2016. Global adjoint tomography: first-generation model, Geophysical Journal International, 207, 17391766.

Cesca, S., Heimann, S., Kriegerowski, M., Saul, J. \& Dahm, T., 2017. Moment tensor inversion for nuclear explosions: What can we learn from the 6 January and 9 September 2016 nuclear tests, North Korea?, Seismol. Res. Lett., 88, 300-310.

Chiang, A., Ichinose, G.A., Dreger, D.S., Ford, S.R., Matzel, E.M., Myers, S.C. \& Walter, W., 2018. Moment Tensor Source-Type Analysis for the Democratic People's Republic of Korea-Declared Nuclear Explosions (2006-2017) and 3 September 2017 Collapse Event, Seismol. Res. Lett., 89, 2152-2165.

Covellone, B.M. \& Savage, B., 2012. A quantitative comparison between 1D and 3D source inversion methodologies: Application to the Middle East, Bulletin of the Seismological Society of America, 102, 2189-2199.

Dahm, T., 1996. Relative moment tensor inversion based on ray theory: theory and synthetic tests, Geophysical Journal International, 124, 245-257.

Dreger, D., 1994. Empirical Green's function study of the January 17, 1994 Northridge, California earthquake, Geophys. Res. Lett., 21, 2633-2636.

Duputel, Z., Rivera, L., Kanamori, H. \& Hayes, G., 2012. W phase source inversion for moderate to large earthquakes (1990-2010), Geophys. J. Int., 189, 1125-1147.

Ekström, G., Nettles, M. \& Dziewoński, A., 2012. The global CMT project 2004-2010: Centroid-moment tensors for 13,017 earthquakes, Physics of the Earth and Planetary Interiors, 200, 1-9.

Fichtner, A., Kennett, B.L., Igel, H. \& Bunge, H.-P., 2009. Full seismic waveform tomography for uppermantle structure in the Australasian region using adjoint methods, Geophysical Journal International, $179,1703-1725$.

Ford, S.R., Dreger, D.S. \& Walter, W.R., 2009. Source analysis of the memorial day explosion, Kimchaek, North Korea, Geophys. Res. Lett., 36.

Ford, S.R., Walter, W.R. \& Dreger, D.S., 2012. Event discrimination using regional moment tensors with teleseismic-P constraints, Bulletin of the Seismological Society of America, 102, 867-872. 
Frohlich, C. \& Davis, S.D., 1999. How well constrained are well-constrained T, B, and P axes in moment tensor catalogs?, Journal of Geophysical Research: Solid Earth, 104, 4901-4910.

Hardebeck, J.L. \& Hauksson, E., 2001. Crustal stress field in southern California and its implications for fault mechanics, Journal of Geophysical Research: Solid Earth, 106, 21859-21882.

Hastings, W.K., 1970. Monte Carlo sampling methods using Markov chains and their applications.

Hauksson, E., 1994. State of stress from focal mechanisms before and after the 1992 Landers earthquake sequence, Bull. Seismol. Soc. Am., 84, 917-934.

Hayes, G.P., Wald, D.J. \& Keranen, K., 2009. Advancing techniques to constrain the geometry of the seismic rupture plane on subduction interfaces a priori: Higher-order functional fits, Geochem. Geophys. Geosyst., 10.

Jia, Z., Ni, S., Chu, R. \& Zhan, Z., 2017. Joint inversion for earthquake depths using local waveforms and amplitude spectra of Rayleigh waves, Pure and Applied Geophysics, 174, 261-277.

Jia, Z., Shen, Z., Zhan, Z., Li, C., Peng, Z. \& Gurnis, M., 2020a. The 2018 Fiji Mw 8.2 and 7.9 deep earthquakes: One doublet in two slabs, Earth Planet. Sci. Lett., 531, 115997.

Jia, Z., Wang, X. \& Zhan, Z., 2020b. Multifault Models of the 2019 Ridgecrest Sequence Highlight Complementary Slip and Fault Junction Instability, Geophys. Res. Lett., 47, e2020GL089802.

Kanamori, H. \& Rivera, L., 2008. Source inversion ofWphase: speeding up seismic tsunami warning, Geophys. J. Int., 175, 222-238.

Kennett, B.L., Engdahl, E. \& Buland, R., 1995. Constraints on seismic velocities in the Earth from traveltimes, Geophysical Journal International, 122, 108-124.

Kikuchi, M. \& Kanamori, H., 1991. Inversion of complex body waves-III, Bull. Seismol. Soc. Am., 81, 23352350.

Lay, T., Burdick, L. \& Helmberger, D.V., 1984. Estimating the yields of the Amchitka tests by waveform intercorrelation, Geophysical Journal International, 78, 181-207.

Lee, E.J., Chen, P., Jordan, T.H., Maechling, P.B., Denolle, M.A. \& Beroza, G.C., 2014. Full-3-D tomography for crustal structure in southern California based on the scattering-integral and the adjoint-wavefield methods, Journal of Geophysical Research: Solid Earth, 119, 6421-6451.

Liu, H., Gurnis, M., Leng, W., Jia, Z. \& Zhan, Z., 2021. Tonga Slab Morphology and Stress Variations Controlled by a Relic Slab: Implications for Deep Earthquakes in the Tonga-Fiji Region, Geophys. Res. Lett., 48, e2020GL091331.

Minson, S.E. \& Dreger, D.S., 2008. Stable inversions for complete moment tensors, Geophys. J. Int., 174, 585-592.

Ni, S., Helmberger, D. \& Pitarka, A., 2010. Rapid source estimation from global calibrated paths, Seismological Research Letters, 81, 498-504.

Plourde, A.P. \& Bostock, M.G., 2019. Relative moment tensors and deep Yakutat seismicity, Geophysical Journal International, 219, 1447-1462.

Rodgers, A.J., Petersson, N.A. \& Sjogreen, B., 2010. Simulation of topographic effects on seismic waves from shallow explosions near the North Korean nuclear test site with emphasis on shear wave generation, Journal of Geophysical Research: Solid Earth, 115.

Savage, B., Morency, C., Covellone, B.M., Rodgers, A. \& Tromp, J., 2014. Short-period, anelastic and anisotropic, waveform-based 3D Middle East model to improve nuclear explosion monitoringRHODE ISLAND UNIV KINGSTON KINGSTON United States.

Simmons, N.A., Myers, S.C., Johannesson, G. \& Matzel, E., 2012. LLNL-G3Dv3: Global P wave tomography model for improved regional and teleseismic travel time prediction, Journal of Geophysical Research: 
Solid Earth, 117.

Tan, Y. \& Helmberger, D., 2007. A new method for determining small earthquake source parameters using short-period P waves, Bull. Seismol. Soc. Am., 97, 1176-1195.

Tao, K., Grand, S.P. \& Niu, F., 2017. Full-waveform inversion of triplicated data using a normalizedcorrelation-coefficient-based misfit function, Geophys. J. Int., 210, 1517-1524.

Tape, C., Liu, Q., Maggi, A. \& Tromp, J., 2009. Adjoint tomography of the southern California crust, Science, 325, 988-992.

Tape, W. \& Tape, C., 2012. A geometric setting for moment tensors, Geophysical Journal International, 190, 476-498.

Tape, W. \& Tape, C., 2013. The classical model for moment tensors, Geophys. J. Int., 195, 1701-1720.

Tape, W. \& Tape, C., 2015. A uniform parametrization of moment tensors, Geophys. J. Int., 202, 2074-2081.

Tarantola, A., 2005. Inverse problem theory and methods for model parameter estimation, edn, Vol., pp. Pages, SIAM.

Vallée, M., 2007. Rupture properties of the giant Sumatra earthquake imaged by empirical Green's function analysis, Bull. Seismol. Soc. Am., 97, S103-S114.

Vavryčuk, V. \& Kim, S.G., 2014. Nonisotropic radiation of the 2013 North Korean nuclear explosion, Geophys. Res. Lett., 41, 7048-7056.

Voytan, D.P., Lay, T., Chaves, E.J. \& Ohman, J.T., 2019. Yield estimates for the six north Korean nuclear tests from teleseismic P wave modeling and intercorrelation of P and Pn recordings, Journal of Geophysical Research: Solid Earth, 124, 4916-4939.

Waldhauser, F. \& Ellsworth, W.L., 2000. A double-difference earthquake location algorithm: Method and application to the northern Hayward fault, California, Bull. Seismol. Soc. Am., 90, 1353-1368.

Wang, D. \& Hutko, A.R., 2018. Relative relocations of the North Korean nuclear tests from 2006 to 2017 using the Hi-Net array in Japan, Geophysical Research Letters, 45, 7481-7487.

Wang, X. \& Zhan, Z., 2020a. Moving from 1-D to 3-D velocity model: automated waveform-based earthquake moment tensor inversion in the Los Angeles region, Geophysical Journal International, 220, 218-234.

Wang, X. \& Zhan, Z., 2020b. Seismotectonics and Fault Geometries of the 2019 Ridgecrest Sequence: Insight From Aftershock Moment Tensor Catalog Using 3-D Green's Functions, Journal of Geophysical Research: Solid Earth, 125, e2020JB019577.

Xu, H., Ni, S., Liu, W., Zhu, H. \& Wang, X., 2020. Focal mechanisms of the 2017 North Korean nuclear test and its early collapse event, Geophys. J. Int., 220, 737-752.

Yang, T., Gurnis, M. \& Zhan, Z., 2017. Trench motion-controlled slab morphology and stress variations: Implications for the isolated 2015 Bonin Islands deep earthquake, Geophys. Res. Lett., 44, 6641-6650.

Zhan, Z., Helmberger, D., Simons, M., Kanamori, H., Wu, W., Cubas, N., Duputel, Z., Chu, R., Tsai, V.C. \& Avouac, J.-P., 2012. Anomalously steep dips of earthquakes in the 2011 Tohoku-Oki source region and possible explanations, Earth Planet. Sci. Lett., 353, 121-133.

Zhang, M. \& Wen, L., 2013. High-precision location and yield of North Korea's 2013 nuclear test, Geophysical Research Letters, 40, 2941-2946.

Zhang, M. \& Wen, L., 2015. Seismological evidence for a low-yield nuclear test on 12 May 2010 in North Korea, Seismological Research Letters, 86, 138-145.

Zhao, L.S. \& Helmberger, D.V., 1994. Source estimation from broad-band regional seismograms, Bulletin of the Seismological Society of America, 84, 91-104.

Zhu, L. \& Ben-Zion, Y., 2013. Parametrization of general seismic potency and moment tensors for source 


\section{inversion of seismic waveform data, Geophysical Journal International, 194, 839-843.}

Zhu, L. \& Helmberger, D.V., 1996. Advancement in source estimation techniques using broadband regional seismograms, Bulletin of the Seismological Society of America, 86, 1634-1641.

Zhu, L. \& Rivera, L.A., 2002. A note on the dynamic and static displacements from a point source in multilayered media, Geophys. J. Int., 148, 619-627. 


\section{ACKNOWLEDGMENTS}

Z. J. and Z. Z. thank and commemorate Don Helmberger for his advising and assistance. We thank

637 the Global Seismic Network (GSN), the International Federation of Digital Seismograph Networks

638 (FDSN), the French Global Network (G), and the Japan Meteorological Agency Seismic Network

639 (JP) for collecting the seismic data, and thank the Incorporated Research Institutions for Seismology

640 (IRIS) for providing public access to them. We thank editor Carl Tape, assistant editor Fern Storey,

641 reviewer Alexandre Plourde, and two other anonymous reviewers for their valuable comments. We

642 thank Jack Muir for helpful discussions. This work is supported by Air Force Research Laboratory

643 (AFRL) Grant FA9453-18-C-0058.

644

645 DATA AVAILABILITY STATEMENT

646 All the data used in our study can be downloaded from the IRIS Data Management Center

647 (http://ds.iris.edu/wilber3/find_event). 